\title{
Patent Foramen Ovale and Thromboembolic Complications
}

\author{
Giovanni Fazio*, Giovanni Ferro, Giuseppe Barbaro, Kalil Fattouch, Filippo Ferrara, Giuseppina Novo and \\ Salvatore Novo
}

Cardiology Unit, Department of Medical Pathophysiology, University La Sapienza, Rome, Palermo, Italy

\begin{abstract}
The foramen ovale, an atrial septal defect, which is essential in the fetal circulation, remains patent through adulthood in approximately $25 \%$ of the general population and therefore it represents the most common persistent abnormality of fetal origin. Patent foramen ovale (PFO) allows interatrial right-to-left blood shunting during those periods of the cardiac cycle in which the right atrial pres-
\end{abstract} sure exceeds the left one.

An increasing number of pathological manifestations of PFO has been recently identified; among these, paradoxical systemic embolism, refractory hypoxemia in patients with right ventricular myocardium infarction or severe pulmonary disease, orthostatic oxygen desaturation in the rare platypnea-orthodeoxia syndrome, neurological decompression illness in divers, high altitude pilots and astronauts, and finally, migraine headache with aura.

Nowadays, many techniques allow to detect a PFO. In this study, we investigated each of them, assessing their potential diagnostic role even in comparison with the main features of the other methods.

Keywords: Patent foramen ovale, diagnostic methods, three dimensional echocardiography.

\section{INTRODUCTION}

The patent foramen ovale (PFO) is a congenital heart defect caused by a non-fusion of the two interatrial fetal septa (septum primum and septum secundum). This anatomic variant may be found in approximately $10 \%$ of the healthy adult population [1-3] and therefore it REPRESENTS the most common persistent abnormality of fetal origin. Patent foramen ovale (PFO) allows interatrial right-to-left blood shunting during those periods of the cardiac cycle in which the right atrial pressure exceeds the left one [27]. This anatomic defect has been recently associated with an increasing number of pathological manifestations; among these are, paradoxical systemic embolism (which can determinate cryptogenetic ischemia, mostly in young patients), neurological decompression illness in divers, high altitude pilots and astronauts, platypneaorthodeoxia syndrome (characterized by orthostatic oxygen desaturation and dyspnea, followed by a clinostatic increase of the $\mathrm{O} 2$ saturation), and finally, migraine headache with aura.

In patients under 55 years old, up to $40 \%$ of ischemic strokes is classified as cryptogenetic strokes, that is, strokes without evidence of an cardiac or epiaortic emboligenic source and with a lesion distribution which does not suggest intracranic small vessels disease [4-9].

A causal relationship between PFO and brain ischemia is still uncertain, but many studies showed a statistical significant link between PFO prevalence or/and interatrial septum aneurism and cryptogenetic ictus, in comparison to the control arm [3-13].

Recent researches also showed the presence of recurrent headache in patients with PFO and right-to-left shunt: the pathogenetic hypothesis of this phenomenon seems to be linked to the genesis of paradoxical emboli which produce microischemia areas inducing headache [4-9, 13-18].

Because of these new pathophisiological hypothesis, the research of a PFO extended to a larger part of population than in the past and this requires the identification of diagnostic methods which would be not-expansive, not-invasive and, at the same time, highly sensitive and specific.

*Address correspondence to this author at the Via Albiri 3 a 90125 Palermo, Italy; Tel: 3334439962; E-mail: Giovanni.fazio-aaaa@poste.it

$1381-6128 / 10 \$ 55.00+.00$

\section{TRANSTHORACIC ECHOCARDIOGRAPHY}

Transthoracic ultrasound is a not-expansive diagnostic method which can be indiscriminately applied to all patients, but it has a low sensitivity [4-13]. Actually, it allows to detect, by doppler velocimetric method, an IAS shunt only in those patients in which the defect is large, but leaving to operator a diagnostic doubt in the rest of individuals.

Other important limits of this technique are its inability to determinate the shunt direction (from left to right or vice versa) and the quality of patient's ultrasound window (Fig. 1).

Vein contrast injection (shaken saline solution) during the performance of the ecocardiographic examination can increase its sensitivity.

\section{TRANSCRANIC ULTRASONOGRAPHY}

Transcranic ultrasonography is a diagnostic method which nowadays represents a fascinating alternative to transthoracic ultrasound in detecting PFO.

It allows to visualize the passage of air microbubbles in the cerebral arterial circle after injection of shaken physiologic solution into a peripheral vein [19], and therefore it indirectly shows the presence of a right-to-left blood shunting. Its sensitivity is comparable to the transesophageal ecocardiography's one, but transcranic ultrasonography does not allow to distinguish the intracardiac shunts from the extracardiac ones.

At cerebral media artery or temporal artery level, microbubbles can be observed and counted out one by one, during their passage.

The recorded signal associated to this passage can individuate the dimension of the defect. To increase the sensitivity of the examination, patient is requested to perform the Valsalva maneuver, so that the venous return to the heart increases, the resistance offered to blood ejection from pulmonary vessels enhances and, subsequently, the septum secundum can grow away from the septum primum, making the shunt more visible [20].

Some method-principles have to be pointed out: the Valsalva maneuver must be performed about 5', after the contrast injection, because it was demonstrated that a solution injected into a peripheral vein requires almost this time to reach the heart: thus, it would be non-sense to perform the maneuver earlier or even before inject- 


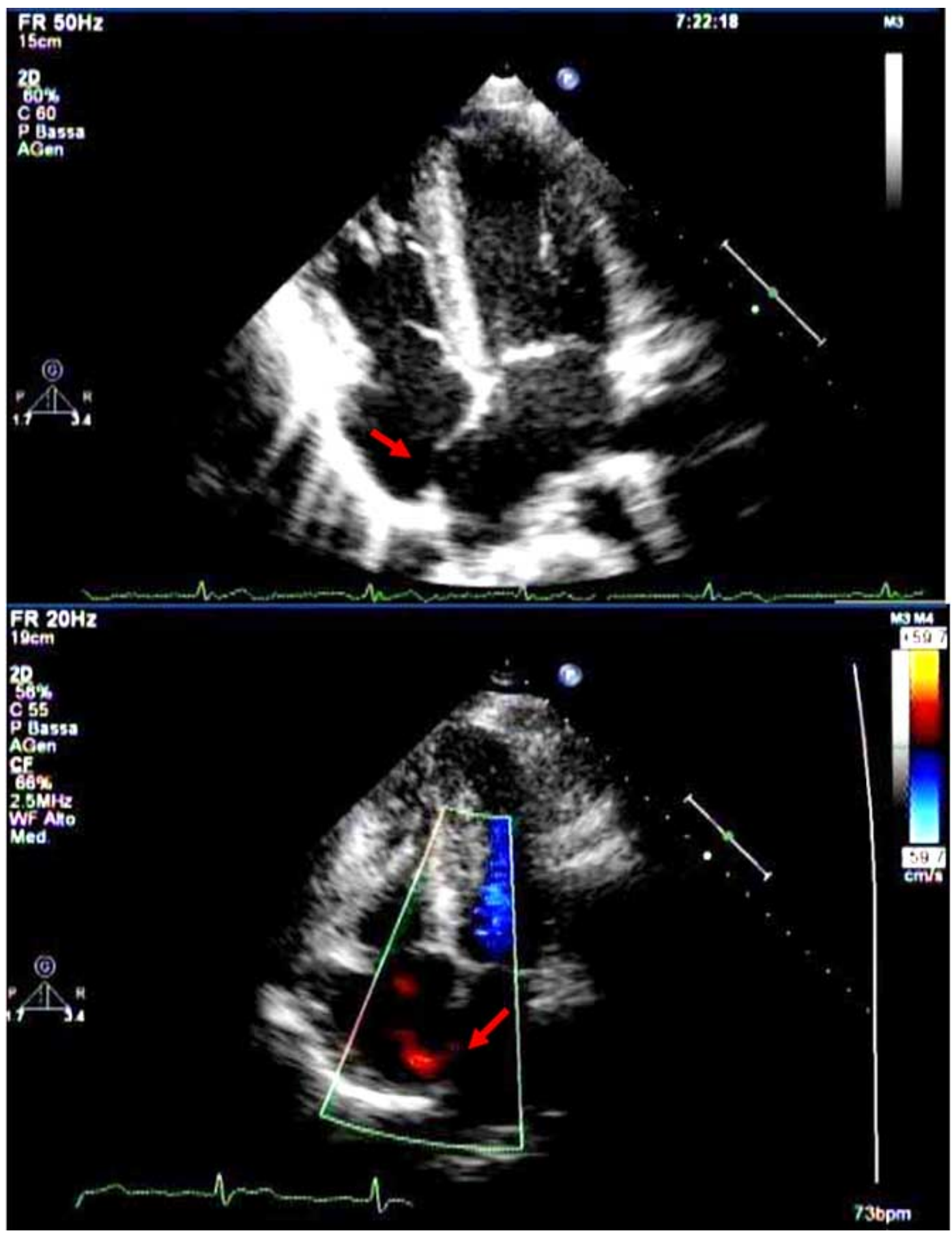

Fig. (1). Transthoracic ultrasound in a patient affected by PFO.

It is possible to detect as the IAS's central part has become more thin then normal, level with the fossa ovalis, with bloodstream going through the same interatrial septum.

ing the shaken physiological solution. On the contrary, it remains still uncertain which is the actual latency time spent by microbubbles to reach the temporal artery, meaning that the examination result is positive: 3 cardiac cycles, 10 seconds or 20 seconds?

In literature, the sensitivity of the transcranic ultrasonography varies from $63 \%$ to about $90 \%$.

It was demonstrated that the best results in terms of sensitivity and specificity can be obtained when the examination is repeated at least twice, the operator waits for at least 40 seconds the possible passage of microbubbles and any threshold value is not considered about the number of microbubbles eventually visualized [21].

However, the specificity of this technique is not high and therefore, in our practice experience, we use it as a screening method (Fig. 2). 


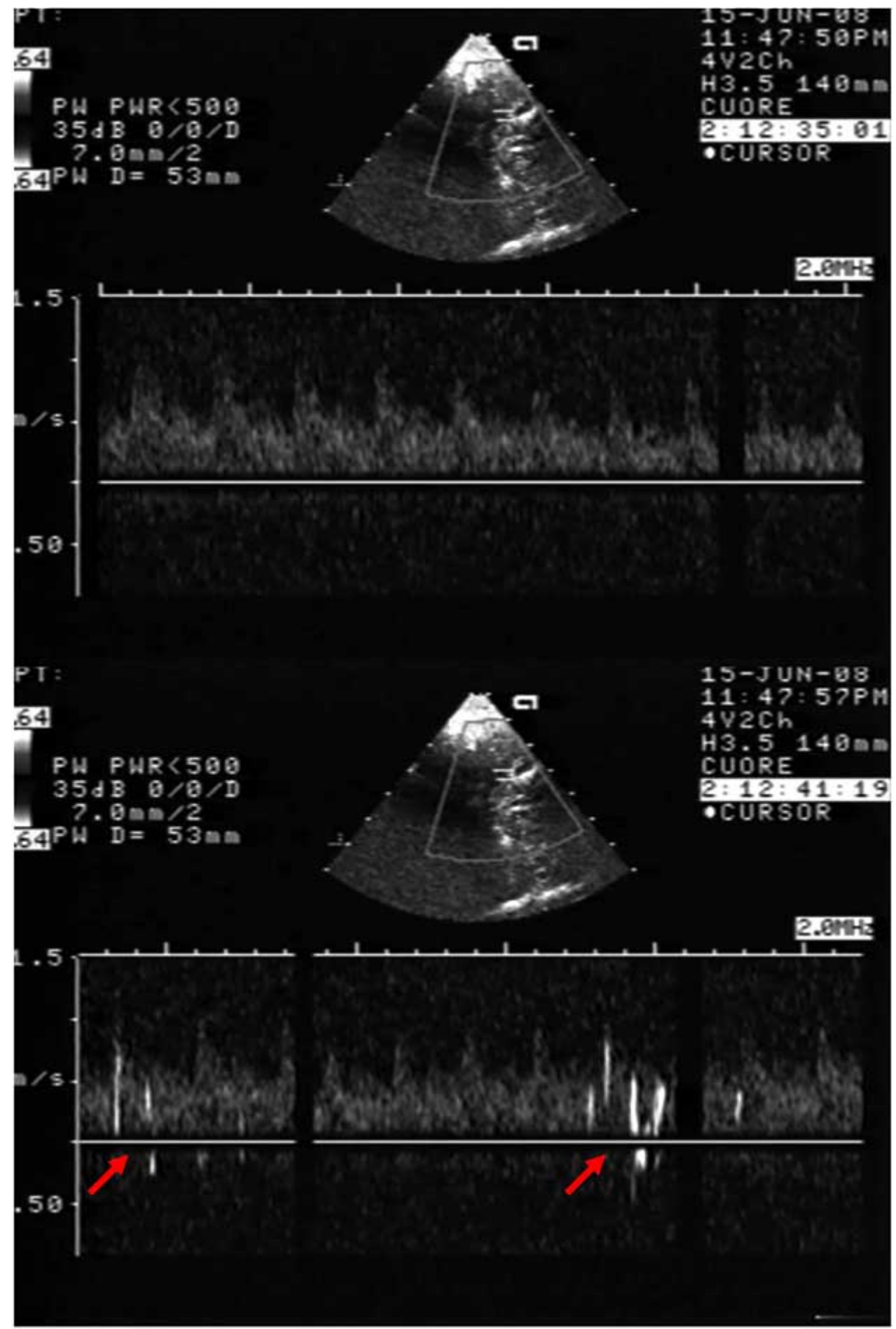

Fig. (2). Transcranic ultrasonography.

It is possible to notice in the lower panel the presence of air microbubbles altering the Doppler spectrum of the upper panel. These microbubbles appeared about 10 "' after injection of a shaken saline solution in a peripheral vein.

\section{TRANSESOPHAGEAL ECHOCARDIOGRAPHY WITH OR WITHOUT CONTRAST}

Transesophageal echocardiography is a high-sensitivity and specificity diagnostic method therefore it allows a more accurate anatomic and functional characterization of the interatrial septum and foramen ovale than the other echocardiographic techniques de- scribed so far, sometimes evidencing already at baseline a nonfusion between the septum primum and the septum secundum.

The use of color-doppler technique makes this method highly sensible by evidencing the right-to-left shunt as a slower flow through the interatrial septum, even if this abnormality can be detected only in $30 \%$ of cases [17-19]. 
During the transesophageal echocardiography, it is possible to perform the Valsalva maneuver, so that the blood pressure increases in the right atrium and the shunt becomes more visible at colordoppler evaluation.

Moreover, this examination overcomes the obstacle of a lowquality ultrasound window but, because it is invasive, not all patients accept it (Fig. 3).

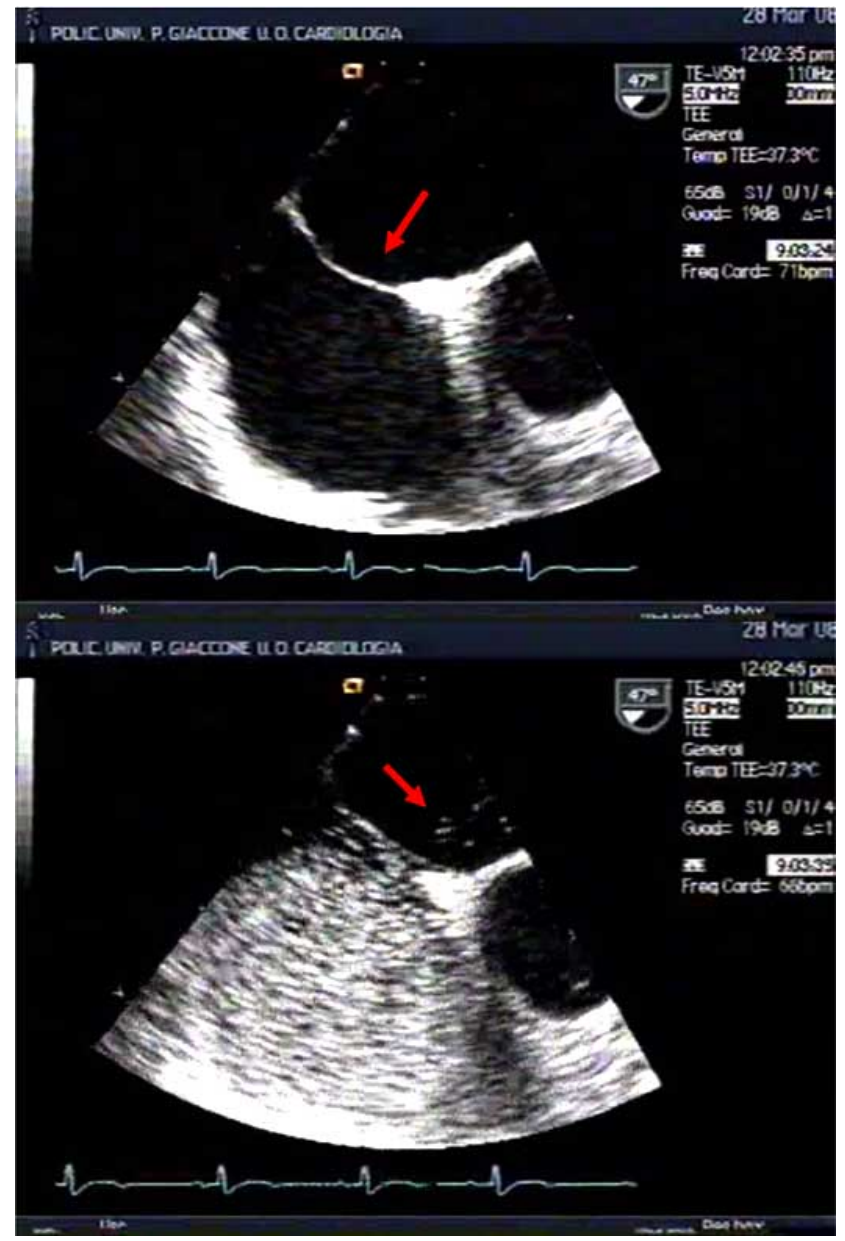

Fig. (3). Image of an interatrial septum in a transesophageal exam performed at baseline (upper panel) and after injection of shaken physiologic solution (lower panel).

In the second panel it is possible to notice an abnormal passage of contrast medium into the left atrium, after opacification of the right one.

\section{CONTRAST ECHOCARDIOGRAPHY}

By this method, after injecting a shaken physiologic solution (contrast medium), PFO can be detected if microbubbles are visualized in the left heart chambers within three cardiac cycles from the full opacification of the right atrium [18].

Contrast saline injection increases the sensitivity of the examination and it may be accompanied by performance of the Valsalva maneuver. The choice of the contrast injection site has also a the diagnostic relevance: in most cases, a venous access sited in the superficial venous circle of the upper limb is chosen, so that contrast medium is injected into the superior vena cava territory.

In this way, as soon as bloodstream comes in the right atrium, it heads for the foramen ovale, favoring identification of the possible right-to-left shunt, therefore, increasing the sensitivity of the diagnostic technique.

The sensitivity of this method resulted very low (about 64\%) in many studies carried out about 10 years ago by different Authors; indeed, in a more recent sensitivity assessment, it was referred equal to $90 \%$, with a specificity of $95 \%$ [18]

Variations in sensitivity and specificity likely depend both on operator's ability / experience and technical difficulty in visualizing images useful for the diagnosis, mostly in patients with a very bad ultrasound window (Fig. 4).

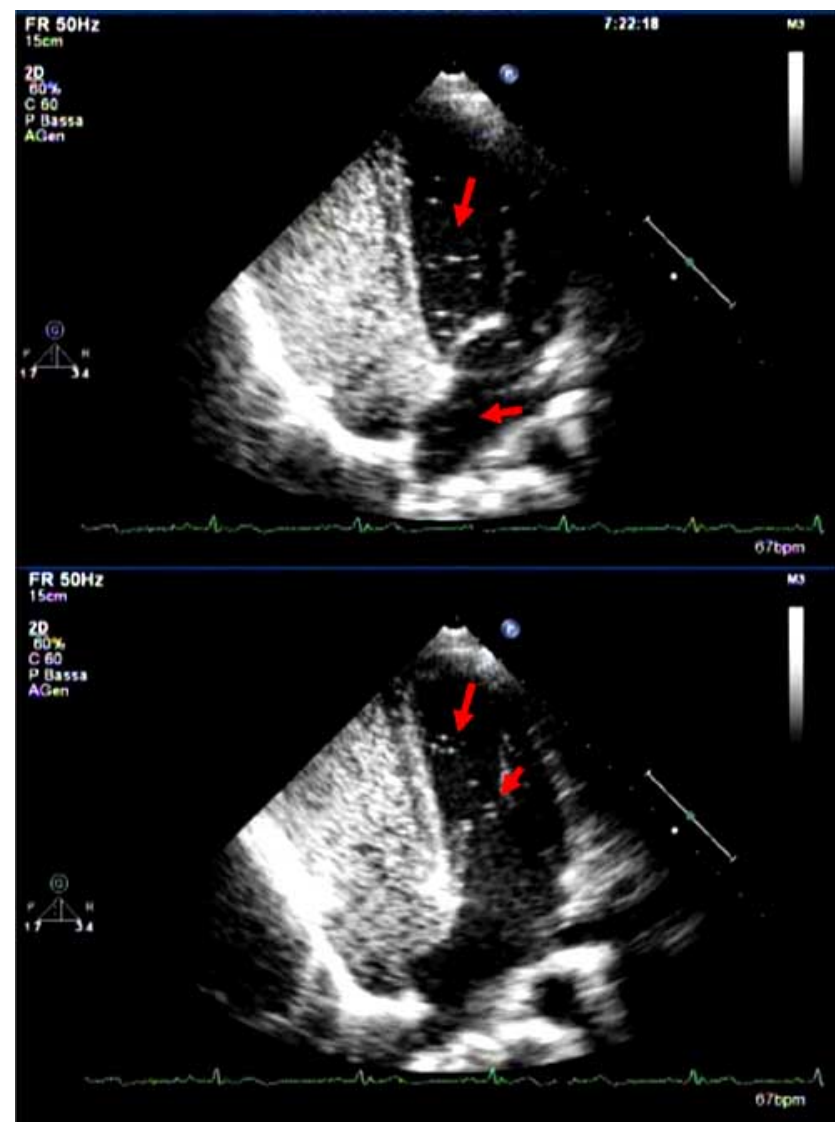

Fig. (4). Two frames in succession of a transthoracic ultrasound performed after injection of shaken saline solution in a patient with PFO.

It is possible to detect an abnormal presence of microbubbles in the left heart within a few seconds from the contrast injection in a peripheral vein.

\section{COMPUTED TOMOGRAPHY (TC)}

This examination allows visualizing PFO only after injection of a contrast medium, thanks to the early opacification of the left atrium $[22,23]$. It has neither higher sensitivity nor higher specificity then the transesophageal echocardiography and it requires a considerable quantity of ionizing radiations: therefore, nowadays it is not used as a diagnostic method.

\section{NUCLEAR MAGNETIC RESONANCE (NMR)}

Till a few years ago, NMR was not employed in the diagnosis of PFO because of its low temporal and spatial resolution capacity; transesophageal echography certainly represented the most accurate technique, but this allowed the heart visualization during the passage of contrast medium along an only plain. After injection of the contrast solution, NMR seems able to detect an interatrial shunt by 
opacificating the left atrium before the pulmonary vein [22-27] (Fig. 5).

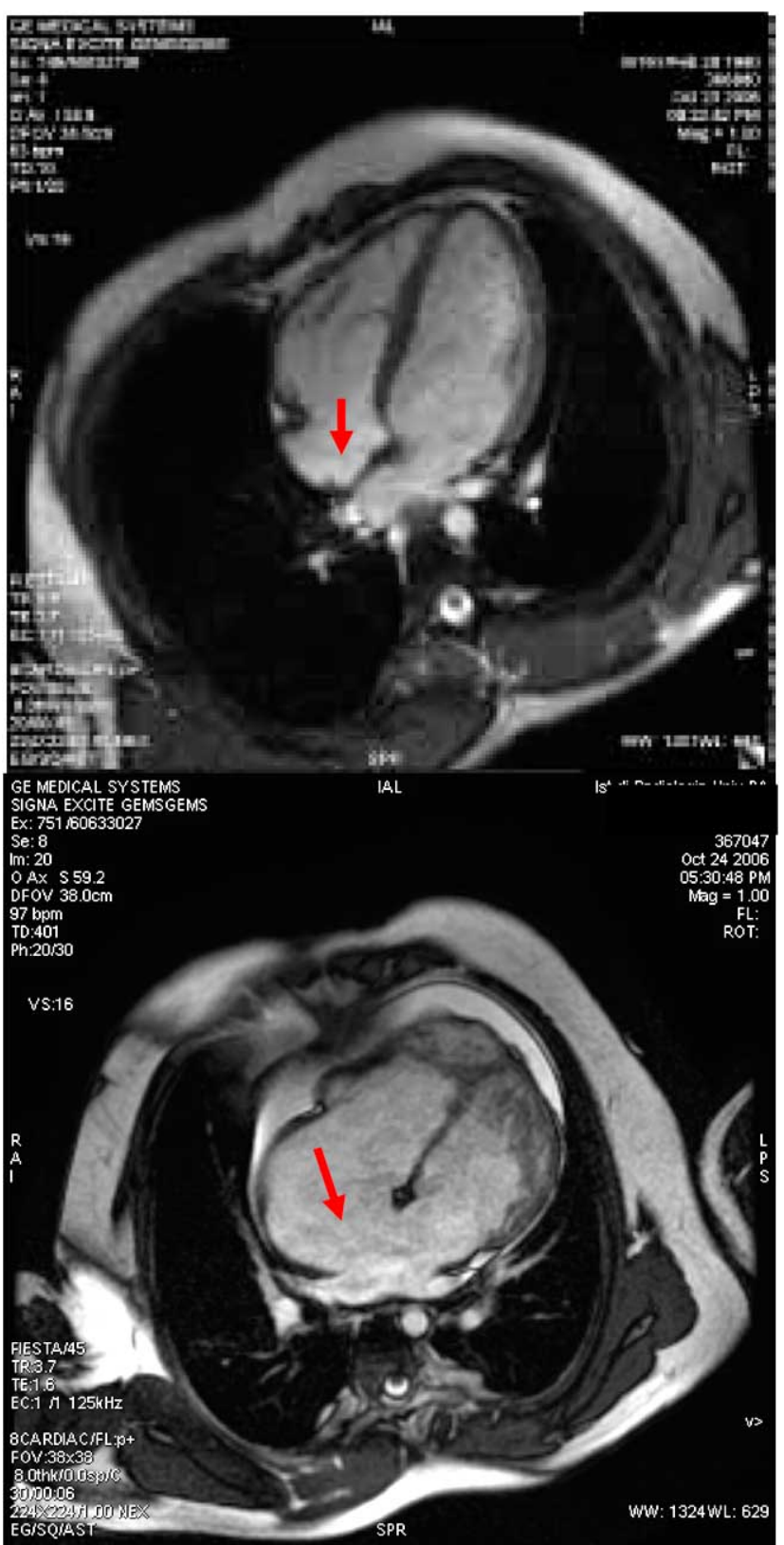

Fig. (5). Anatomical images in a NMR performed without contrast medium. The upper panel shows a PFO while the lower one shows a large interatrial defect causing a remarkable right-to-left shunt and a dilatation of the right atrium. The accurate anatomical resolution of this diagnostic technique at baseline allows to visualize perfectly the interatrial septum.

This diagnostic method is still scarcely employed to detect PFO and in the future more accurate images will be likely obtained by using techniques which allow a three-dimensional study of the heart.

\section{3-DIMENSIONAL ECHOCARDIOGRAPHY}

3-dimensional echocardiography offers many different advantages, compared with usual echocardiographic techniques.
First of all, it allows to make a diagnosis already at baseline (without the use of contrast medium), because it guarantees a detailed reconstruction of the heart by using all the three spatial dimensions; moreover, it is possible to perform the examination in real time or to revise images afterwards, sparing patient long and exhausting diagnostic meetings. During reconstruction, this method allows to analyze separately each of the 3 spatial dimensions, assessing movements of the interatrial septum during both systole and diastole.

Because of the high specificity of this method, even if any study has not been published in the literature as yet, on the basis of our own experience, we think 3-dimensional echocardiography will be the "gold standard" diagnostic technique for detecting PFO in the future. Actually, it does not need any venous access and subsequently, it does not require at least two operators, unlike the contrast-enhanced instrumental methods; moreover, because it uses a transthoracic probe, 3-dimensional echocardiography is not invasive, and so it is more tolerated by patient then the transesophageal ultrasound.

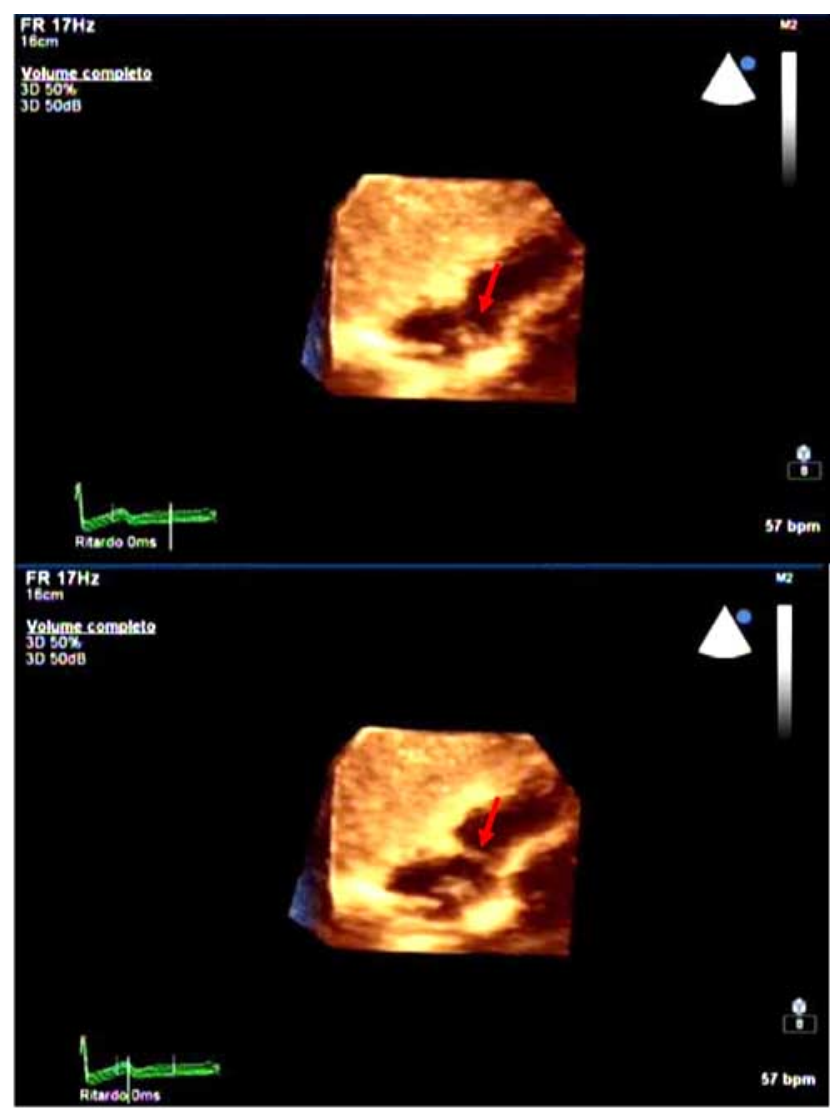

Fig. (6). Post-processing reconstruction of a 3-dimensional transthoracic ultrasound.

It is possible to visualize an abnormal movement of the IAS toward the left atrium: the septum is not visible in the first frame (upper panel) and it appears in the second one (lower panel). The two frames were performed in succession, with an interval of about $600 \mathrm{~ms}$.

The only limit of this method is the image quality, especially in patients with a suboptimal echocardiography window, but there is no doubt that it can be significantly improved thanks to the use of a 3-dimensional probe (Fig. 6). Anyway, it is possible to increase the sensitivity of 3-dimensional echocardiography by injecting a con- 
trast medium (shaken saline solution) to visualize directly in a three-dimensional way and the passage of air microbubbles through a PFO.

\section{REFERENCES}

References 27-29 are related articles recently published.

[1] Hara H, Virmani R, Ladich E. Patent foramen ovale: current pathology, pathophysiology, and clinical status. J Am Coll Cardiol 2005; 46 (9): 1768-76.

[2] Lucas JF, Radtke WA, Bandisode VM, Fairbrother DL, Shirali GS. Characteristic of the interatrial communication in patients undergoing trancatheter device closure of atrial septal defects for cryptogenic stroke. Echocardiography 2005; 22(10): 814-7.

[3] Hausmann D, Mugge A, Daniel WG. Identification of patent foramen ovale permitting paradoxic embolism. J Am Coll Cardiol 1995; 26: 1030-8.

[4] Wu LA, Malouf JF, Dearani JA. Patent foramen ovale in cryptogenic stroke: current understanding and management options. Arch Intern Med 2004; 164: 950-6.

[5] Wahl A, Windecker S, Meier B. Patent foramen ovale: pathophysiology and therapeutic options in symptomatic patients. Minerva Cardioangiol 2001; 49(6): 403-11. Review.

[6] Schrader R. Indication and techniques of transcatheter closure of patent foramen ovale. J Interven Cardiol 2003; 16: 543-51.

[7] Sacco RL, Ellemberg JH, Mohr JP, Tatemichi TK, Hier DB, Price TR. Infarcts of undetermined cause: the NINCDS stroke data bank. Ann Neurol 1989; 25: 382-90.

[8] Shunichi Homma, Ralph L. Sacco patent foramen ovale and stroke. Circulation 2005; 112; 1063-72

[9] Kerut EK, Norfleet WT, Plotnick GD, Giles TD. Patent foramen ovale: a review of associated conditions and the impact of physiological size. J Am Coll Cardiol 2001; 38: 613-23.

[10] Cohnheim J. Thrombosis and embolism. Ann Clin Cardiol 1997; 134-7.

[11] Wilmshurst PT, Ellis BG, Jenkins BS. Paradoxical gas embolism in a scuba diver with an atrial septal defect. Br Med J (Clin Res Ed) 1986; 293: 1277.

[12] Torti SR, Billinger M, Schwerzmann M, et al. Risk of decompression illness among 230 divers in relation to the presence and size of patent foramen ovale. Eur Heart J 2004; 25: 1014-20.

[13] Steiner MM, Di Tullio MR, Rundek T. Patent foramen ovale size and embolic brain imaging findings among patients with ischemic stroke. Stroke 1998; 29: 944-8.

[14] Bogousslavsky J, Garazi S, Jeanrenaud X, Aebischer N, Van Melle G. Stroke recurrence in patients with patent foramen ovale: the lausanne study - lausanne stroke with paradoxal embolism study group. Neurology 1996; 46: 1301-5.

[15] Mas JL, Zuber M. Recurrent cerebrovascular events in patients with patent foramen ovale, atrial septal aneurysm, or both and cryptogenic stroke or transient ischemic attack: French Study Group on Patent Foramen Ovale and Atrial Septal Aneurysm. Am Heart J 1995; 130: 1083-8.

[16] De Castro S, Cartoni D, Fiorelli M. Patent foramen ovale and its embolic implications. Am J Cardiol2000; 86: 51G -2.

[17] Belkin RN, Pollack BD, Ruggiero ML, Alas LL, Tatini U. Comparison of transesophageal and transthoracic echocardiography with contrast and color flow Doppler in the detection of patent foramen ovale. Am Heart J 1994; 128: 520-5.

[18] Chen W-J, Kuan P, Lien W-P, Lan F-Y. Detection of patent foramen ovale by contrast transesophageal echocardiography. Chest 1992; 101; 1515-20.

[19] Daniëls C, Weytjens C, Cosyns B, et al. Second harmonic transthoracic echocardiography: the new reference screening method for the detection of patent foramen ovale. Eur J Echocardiogr 2004; 5: $449 \mathrm{e}-452$.

[20] Scacciatella P, Solaro C, Fava A, et al. Marra Il forame ovale pervio in pazienti con stroke criptogenetico: fisiopatologia, diagnosi e trattamento. Cardiol Ambulat 2006; 2: 93-100.

[21] Zanette EM, Mancini G, De Castro S, et al. Patent foramen ovale and transcranial doppler comparison of different procedures. Stroke 1996; 27: 2251-5.

[22] Droste DW, Silling K, Stypmann J, et al. Bernd Ringelstein. Contrast transcranial doppler ultrasound in the detection of right-to-left shunts time window and threshold in microbubble numbers. Stroke 2000; 31: 1640-5.

[23] Saremi F, Attai SF, Narula J. 64 multidetector CT in patent foramen ovale. Heart 2007; 93; 505

[24] Pohost GM, Hung L, Doyle M. Clinical use of cardiovascular magnetic resonance. Circulation 2003; 108: 647-53.

[25] Lamy C, Giannesini C, Zuber M, et al. Clinical and imaging findings in cryptogenic stroke patients with and without patent foramen ovale: the PFO-ASA study - atrial septal aneurysm. Stroke 2002; 33: 706 .

[26] Mohrs OK, Petersen SE, Erkapic D, et al. Diagnosis of patent foramen ovale using contrast-enhanced dynamic MRI: A Pilot Study. Am J Roentgenol 2005; 184(1): 234-40.

[27] Mongiovi M, Pipitone S. Supraventricular tachycardia in fetus: how can we treat? Curr Pharm Des 2008; 14(8): 736-42.

[28] Karpawich PP, Pettersen MD, Gupta P, Shah N. Infants and children with tachycardia: natural history and drug administration. Curr Pharm Des 2008; 14(8): 743-52.

[29] Picano E, Pasanisi E, Venneri L, Agrusta M, Mottola G, Sicari R. Stress echocardiography. Curr Pharm Des 2005; 11(17): 2137-49. 